\title{
Structure of ATP synthase under strain during catalysis
}

\author{
Hui Guo ${ }^{1,2}$ and John L. Rubinstein ${ }^{1,2,3}$
}

1. Molecular Medicine Program, The Hospital for Sick Children, Toronto, Ontario, Canada

2. Department of Medical Biophysics, The University of Toronto, Ontario, Canada

3. Department of Biochemistry, The University of Toronto, Ontario, Canada

* Correspondence: john.rubinstein@utoronto.ca

\begin{abstract}
ATP synthases are macromolecular machines consisting of an ATP-hydrolysis-driven $\mathrm{F}_{1}$ motor and a proton-translocation-driven $F_{O}$ motor. The $F_{1}$ and $F_{O}$ motors oppose each other's action on a shared rotor subcomplex and are held stationary relative to each other by a peripheral stalk. Structures of resting mitochondrial ATP synthases revealed a left-handed curvature of the peripheral stalk even though rotation of the rotor, driven by either ATP hydrolysis in $\mathrm{F}_{1}$ or proton translocation through $\mathrm{F}_{\mathrm{O}}$, would apply a right-handed bending force to the stalk. We used cryoEM to image yeast mitochondrial ATP synthase under strain during ATP-hydrolysis-driven rotary catalysis, revealing a large deformation of the peripheral stalk. The structures show how the peripheral stalk opposes the bending force and suggests that proton translocation during ATP synthesis causes accumulation of strain in the stalk, which relaxes by driving the relative rotation of the rotor through six sub-steps within $\mathrm{F}_{1}$, leading to catalysis.
\end{abstract}




\section{Introduction}

ATP synthases use a transmembrane electrochemical proton motive force $(\mathrm{pmf})$ to generate adenosine triphosphate (ATP) from adenosine diphosphate (ADP) and inorganic phosphate (Pi). The enzyme complex consists of two molecular motors positioned to oppose each other's action on a shared rotor subcomplex (Fig. 1A, left). The membrane-embedded Fo motor is driven by proton translocation across the membrane through two offset half channels (Junge et al., 1997; Vik and Antonio, 1994) while the soluble $F_{1}$ motor is powered by ATP hydrolysis. In Saccharomyces cerevisiae, the $\mathrm{F}_{\mathrm{O}}$ region contains subunits a, e, f, g, i/j, k, 8, part of subunit b, and the $\mathrm{c}_{10}$-ring of the rotor (Liu et al., 2015), while the $\mathrm{F}_{1}$ region includes a trimer of catalytic subunit $\alpha \beta$ pairs and subunits $\gamma, \delta$, and $\varepsilon$ from the rotor (Abrahams et al., 1994). Coupling between $\mathrm{F}_{1}$ and $\mathrm{F}_{\mathrm{O}}$ requires that the two motors are held stationary relative to each other by a peripheral stalk subcomplex (Fig. 1A, green structure), which in yeast is formed from subunits b, $\mathrm{d}, \mathrm{h}$, and OSCP (the oligomycin sensitivity conferral protein).

During ATP synthesis, proton translocation through $\mathrm{F}_{\mathrm{O}}$ at the interface of subunit a and the cring causes the $\gamma \delta \varepsilon \mathrm{c}_{10}$ rotor (Fig. 1A, outlined in black) to turn. Rotation of subunit $\gamma$ within $\mathrm{F}_{1}$ leads each $\alpha \beta$ pairs to cycle through open, tight, and loose conformations that result in the formation of ATP. Conversely, sequential ATP hydrolysis at each of the three $\alpha \beta$ pairs in $F_{1}$ causes the $\gamma$ subunit to turn in the opposite direction, rotating the proton-carrying c-ring against subunit a in $\mathrm{F}_{\mathrm{O}}$ and pumping protons across the membrane. Even with the rotor turning at hundreds of revolutions per second (Bilyard et al., 2013; Kobayashi et al., 2020) there is little or no 'slip' (Soga et al., 2017) and the $\mathrm{H}^{+}$:ATP ratio remains constant. In $S$. cerevisiae this ratio is 10:3 due to the ten proton-carrying c subunits in Fo and three catalytic sites in F1 (Stock et al., 1999). With this $\mathrm{H}^{+}$:ATP ratio, when $10 \times$ the free energy of proton translocation $\left(\Delta \tilde{\mu}_{H^{+}}\right)$is more negative than $3 \times$ the free energy of ATP hydrolysis $\left(\Delta G_{A T P}\right)$ the $\mathrm{F}_{\mathrm{O}}$ motor overpowers the $\mathrm{F}_{1}$ motor, forcing it to synthesize ATP. When $3 \times \Delta G_{A T P}$ is more negative than $10 \times \Delta \tilde{\mu}_{H^{+}}, \mathrm{F}_{1}$ overpowers $F_{O}$ and the enzyme hydrolyzes ATP to pump protons.

Synthesis or hydrolysis of each ATP molecule is associated with a $\sim 120^{\circ}$ rotation of the $\gamma$ subunit within $\mathrm{F}_{1}$, leading to conformations of the enzyme known as rotational State 1, 2, and 3 (e.g. Zhou et al., 2015). During ATP hydrolysis, which is better studied than ATP synthesis, this 
$\sim 120^{\circ}$ rotation is broken down into a $\sim 40^{\circ}$ sub-step as the enzyme transitions from a 'catalytic dwell' to a 'binding dwell', and an $\sim 80^{\circ}$ sub-step as the enzyme transitions to the next catalytic dwell (Yasuda et al., 2001a; Bilyard et al., 2013; Martin et al., 2014; Steel et al., 2015) (Fig. 1B, clockwise). ATP hydrolysis likely occurs during the $\sim 40^{\circ}$ sub-step while ATP binding likely occurs during the $\sim 80^{\circ}$ sub-step (Nishizaka et al., 2004; Adachi et al., 2007; Steel et al., 2015). Consequently, the expected sequence of states for a $360^{\circ}$ rotation of the rotor during ATP synthesis is State $1_{\text {binding }} \rightarrow$ State $1_{\text {catalytic }} \rightarrow$ State $2_{\text {binding }} \rightarrow$ State $2_{\text {catalytic }} \rightarrow$ State $3_{\text {binding }} \rightarrow$ State


proton-translocation steps in Fo suggests that the enzyme cycles between strained and relaxed conformations during catalysis (Cherepanov et al., 1999; Pänke and Rumberg, 1999). Early cryoEM noted that the peripheral stalks of mitochondrial ATP synthases have a left-handed curvature (Lau et al., 2008; Rubinstein et al., 2003) (Fig. 1A, left). However, torque applied to the rotor following proton translocation through $\mathrm{F}_{\mathrm{O}}$ (Fig. 1A, right, blue arrows) would tend to rotate the $\alpha_{3} \beta_{3}$ hexamer in the same direction as the torque, inducing a right-handed curvature of the peripheral stalk as it resists the rotation. Similarly, the opposite torque applied to the opposite end of the rotor by ATP hydrolysis in $\mathrm{F}_{1}$ (Fig. 1A, right, red arrows) would tend to rotate the membrane-embedded region of $F_{O}$ along with the c-ring, also inducing a right-handed curvature of the peripheral stalk as it resists the rotation (Lau et al., 2008). Previously observed structures were obtained in the absence of a pmf or free ATP (Flygaard et al., 2020; Gu et al., 2019; Guo et al., 2019; Hahn et al., 2018, 2016; Lau et al., 2008, p. 20; Mühleip et al., 2021, 2019; Murphy et al., 2019; Pinke et al., 2020; Rubinstein et al., 2003; Sobti et al., 2016; Spikes et al., 2020; Srivastava et al., 2018; Zhou et al., 2015), suggesting that the peripheral stalk may act as a spring that has a left-handed curvature when relaxed but a right-handed curvature under strain during catalysis (Lau et al., 2008).

\section{Results and discussions}

The peripheral stalk shows pronounced bending under strain during ATP hydrolysis

We purified S. cerevisiae ATP synthase with the detergent n-Dodecyl- $\beta$-D-Maltopyranoside (DDM), which results in a monomeric preparation of the enzyme (Rubinstein and Walker, 2002; Srivastava et al., 2018), and determined its structure by cryoEM (Fig. S1 and S2, Supplementary Table 1 and 2). A high-resolution map of the intact complex was generated by combining 
multiple maps from focused refinements (Fig. 1C and S1C). In this map, the peripheral stalk shows the left-handed curvature seen previously. Three-dimensional (3D) classification allowed particle images to be separated into six rotor positions, corresponding to the catalytic and binding dwells for each of the three main rotational states. These conformations resemble recent catalytic and binding dwell structures for an isolated bacterial $F_{1}$ subcomplex imaged during ATP hydrolysis, where the absence of the peripheral stalk resulted in all catalytic dwell structures being identical and all binding dwell structures being identical (Sobti et al., 2021). For yeast ATP synthase imaged without ATP, the catalytic dwell structures show $\alpha \beta_{\text {tight }}$ either in the open conformation lacking nucleotide or in a closed conformation with weak nucleotide density, and the binding dwell structures show $\alpha \beta_{\text {tight }}$ only in an open conformation without nucleotide (Fig. S3). The existence of $\alpha \beta_{\text {tight }}$ in an open conformation without visible nucleotide density is likely an artefact from loss of ATP during the purification of the enzyme. Further classification of the State $1_{\text {catalytic }}$ conformation resulted in classes distinguished by variability in the position of the peripheral stalk and a slight rotation of the rotor relative to subunit a. These classes were

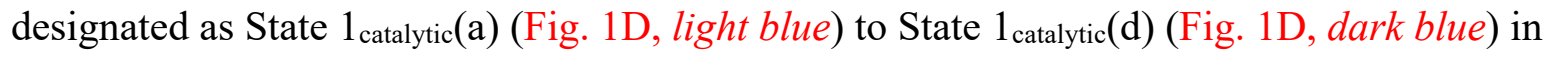
order of increasing straightening of the peripheral stalk (Supplementary Video 1, 'no ATP' condition). As these structures were determined in the absence of free ATP, they likely represent energetically similar conformations that can be reached by thermal fluctuation of the enzyme structure (Murphy et al., 2019; Zhou et al., 2015).

To test the hypothesis that the peripheral stalk of ATP synthase deforms under strain, we next added $10 \mathrm{mM}$ ATP to the preparation and froze cryoEM specimens within $10 \mathrm{~s}$. Analysis of a small dataset of images for this condition collected with a screening electron microscope revealed conformations of the enzyme not seen in the absence of free ATP (Fig. S4). Therefore, a large dataset was collected for the specimen with a high-resolution microscope (Fig. S5). Classification of the resulting dataset yielded maps showing six different $F_{1}$ states, corresponding to the catalytic and binding dwell structures from each of the three main rotational states. Subclassification of these populations separated each catalytic and each binding state into conformations with increasing rotation of the rotor relative to subunit a, and increasingly strained peripheral stalks, labelled as 'a', 'b', 'c', etc. Overall, 27 unique conformations were identified: State $1_{\text {binding }}($ a to $d)$, State $1_{\text {catalytic }}$ (a to h), State $2_{\text {binding }}$ (a to b), State 2 catalytic(a to e), State $3_{\text {binding }}$ (a 


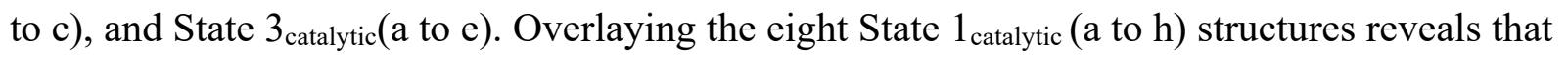
during ATP hydrolysis the peripheral stalk exhibits a large bending motion, transitioning from a left-handed curvature (Fig. 1E, light purple) to the predicted right-handed curvature (Fig. 1E, dark purple; Supplementary Video 1, 'During ATP hydrolysis' condition). Without ATP and during ATP hydrolysis, the left-handed curvature of the peripheral stalk remains the most highly populated conformation of the enzyme (Fig. 1F).

\section{The flexible peripheral stalk accommodates rigid rotation of the rotor during ATP hydrolysis}

To facilitate comparison of the ATP synthase conformations that occur during ATP hydrolysis, backbone models of the protein structure were fit flexibly into each of the 27 maps (Fig. 2A). Remarkably, the $\alpha_{3} \beta_{3} \gamma \delta \varepsilon c_{10}$ models from all 18 catalytic dwell conformations could be overlaid with high-fidelity (Fig. 2B, left), as could the nine $\alpha_{3} \beta_{3} \gamma \delta \varepsilon c_{10}$ models from binding dwell conformations (Fig. 2B, right), with some limited flexibility at the interface between $\mathrm{F}_{1}$ and the c-ring. This observation shows that, other than being in a catalytic or binding dwell conformation, the differences between the structures are mostly due to deformation of the peripheral stalk subunits and the rotation of the c-ring relative to subunit a in Fo. Comparison of the eight State $1_{\text {catalytic }}$ models shows that the $\alpha_{3} \beta_{3} \gamma \delta \varepsilon c_{10}$ rotor can turn $\sim 80^{\circ}$ against subunit a in $F_{O}$, or more than one fifth of a complete revolution, before transition to the next binding dwell conformation (Fig. 2C). Bending of the peripheral stalk and not the central rotor of the complex supports suggestions that the peripheral stalk is the most compliant part of the enzyme and stores energy during rotary catalysis (Guo et al., 2019; Hahn et al., 2018; Murphy et al., 2019; Sobti et al., 2016; Sorgen et al., 1999, 1998; Zhou et al., 2015).

\section{The peripheral stalk bends by deformation of subunits $d, f$, and $h$}

The peripheral stalk of yeast ATP synthase contains subunits b, d, h, and OSCP (Fig. 3A). Although atomic models for subunits $b, d$, and OSCP have been constructed from previous cryoEM of ATP synthase (Srivastava et al., 2018), model quality for the 92-residue subunit $\mathrm{h}$ in earlier structures was low due to flexibility in both the peripheral stalk overall and subunit $h$ specifically. Focused refinement of the peripheral stalk in the current structure resulted in continuous density for most of subunit $\mathrm{h}$, allowing for construction of an atomic model for residues 1 to 62 based on predictions from AlphaFold (Jumper et al., 2021) (Fig. 3A, blue; Fig. 
S6). Interestingly, despite density immediately C-terminal of His62 in subunit h appearing disordered, an additional density that interacts with subunits a, $\mathrm{d}, \mathrm{f}$, and 8 indicates that the $\mathrm{C}$ terminus of the protein reaches the membrane surface, as suggested previously (Rubinstein et al., 2005) (Fig. 3A, dashed box). Therefore, subunit h spans the entire distance from $\mathrm{F}_{1}$ to $\mathrm{F}_{\mathrm{O}}$, a role usually attributed only to subunit $b$, and different from subunit $F_{6}$, the shorter mammalian homologue of subunit h (Spikes et al., 2020).

Aligning the eight structures corresponding to the State $1_{\text {catalytic }}$ by their Fo regions reveals that the dramatic bending of the peripheral stalk is facilitated mainly by deformation of subunits $b, d$, and h (Fig. 3B; Supplementary Video 1, 'During ATP hydrolysis' condition). In conformations that show only slight bending of the peripheral stalk, such as State $1_{\text {catalytic }}(b$ to $d)$, deviation from the relaxed State $1_{\text {catalytic }}$ (a) conformation is mediated primarily by a pivot point in subunits $b$ and $\mathrm{d}$ close to the membrane surface (Fig. 3C, red arrow). In the more strained conformations like State $1_{\text {catalytic }}(\mathrm{h})$, a second pivot point in subunit $\mathrm{b}$ at the top of subunit $\mathrm{d}$ is apparent (Fig. $3 \mathrm{C}$, yellow arrow). The two pivot points are located at either end of subunit d, indicating that subunit $\mathrm{d}$ controls where the peripheral stalk bends and likely acts to oppose the bending force, inducing the left-handed curvature of the peripheral stalk when it is not under strain. The structure of subunit $d$, with an $\alpha$-helical hairpin that allows it to push against subunit $b$, is ideally optimized for its role of applying a force that attempts to restore the relaxed conformation of the peripheral stalk during ATP hydrolysis or synthesis (Fig. 3D; Supplementary Video 1, orange subunit). Subunit h contains two disordered regions close to the two pivot points defined by subunit d, which allows it to withstand the large conformational changes that occurs around the pivot points (Fig. 3D, blue asterisks). In contrast with the spring-like peripheral stalk seen here for the yeast ATP synthase, the unusually large peripheral stalk of algal ATP synthase from Polytomella sp., although imaged in the absence of substrate, appears mostly rigid, with the OSCP subunit that connects the catalytic domain to the rest of the peripheral stalk showing the most flexibility (Murphy et al., 2019).

\section{Overall rotation cycle of yeast ATP synthase}

Despite the presence of a high concentration of ATP in the buffer used for freezing specimens during ATP hydrolysis, State $1_{\text {catalytic }}(\mathrm{a})$, the least strained of the State $1_{\text {catalytic }}$ conformations, 
appears to have MgADP bound in its $\alpha \beta_{\text {tight }}$ site (Fig. 4A, left). In contrast, refinement of the $\mathrm{F}_{1}$ region with particle images combined from State $1_{\text {catalytic }}(\mathrm{e}$ to $\mathrm{h}$ ), the four most strained of the State $1_{\text {catalytic }}$ conformations, resulted in a structure similar to State $1_{\text {catalytic }}($ a) but with what appears to be MgATP bound to $\alpha \beta_{\text {tight }}$ (Fig. 4A, right). In the presence of free ATP, ATP hydrolysis occurs at the $\alpha \beta_{\text {tight }}$ site and MgADP within the site is expected to inhibit this hydrolytic activity. Therefore, the presence of MgADP in $\alpha \beta_{\text {tight }}$ of the non-strained conformation suggests that many of the complexes in this conformation are in the well-known MgADP inhibited state (Abrahams et al., 1994; Bowler et al., 2007). Similarly inactive complexes have been detected previously even in the presence of free ATP (Hirono-Hara et al., 2001; Noji et al., 1997). In contrast, the structures that show the more strained peripheral stalks appear to be calculated from images of active enzyme particles. Density for the binding dwell conformations suggests that they contain MgADP with Pi in the $\alpha \beta_{\text {tight }}$ site (Fig. S7A), as was seen in the bacterial $F_{1}$ region during ATP hydrolysis (Sobti et al., 2021).

To place the 27 conformations of ATP synthase observed during ATP hydrolysis into a rotational sequence, the positions of subunit $\gamma$ relative to $\alpha_{3} \beta_{3}$ in $F_{1}$ (Fig. 4B) and of the c-ring relative to subunit a in $\mathrm{F}_{\mathrm{O}}$ (Fig. 4C) were measured and plotted on circles that represent a $360^{\circ}$ rotation. As described above, the $\alpha_{3} \beta_{3} \gamma \delta \varepsilon c_{10}$ subcomplex is found in three catalytic dwell conformations and three binding dwell conformations, resulting in only six unique positions of subunit $\gamma$ relative to $\alpha_{3} \beta_{3}$ in $F_{1}$ (Fig. 2B). Consistent with the isolated bacterial $F_{1}$ region (Sobti et al., 2021; Yasuda et al., 2001b), ATP hydrolysis in $\alpha \beta_{\text {tight }}$ of the yeast catalytic dwell conformation appears to induce a slightly more open conformation of the $\alpha \beta$ pair and a $\sim 36^{\circ}$ rotation of the rotor (Fig. S7B), leaving the enzyme in a binding dwell. MgADP and Pi are then released from the $\alpha \beta_{\text {tight }}$ site and ATP binding to the $\alpha \beta_{\text {open }}$ site drives an $\sim 84^{\circ}$ rotation of the rotor to the next catalytic dwell conformation. Repetition of this process two more times completes the $360^{\circ}$ rotation cycle for ATP hydrolysis (Fig. 4B, clockwise arrows), while for ATP synthesis the reverse reaction is driven by rotation of the rotor in the opposite direction (Fig. 4B, counter-clockwise arrows).

In contrast to the six unique positions of subunit $\gamma$ relative to $\alpha_{3} \beta_{3}$ in $F_{1}$, there are 27 unique positions of the c-ring relative to subunit a in $\mathrm{F}_{\mathrm{O}}$. Plotting the angle of the c-ring relative to 
subunit a in Fo produces a series of arcs that show the range of rotation of the ring within each catalytic or binding dwell state (Fig. 4C, black, blue, yellow, green, red, and cyan arcs). These arcs reveal that as the c-ring rotates in the ATP hydrolysis direction, each state exhibits a decreasing strain on the peripheral stalk (Fig. 4C, clockwise arrow). For example, for State

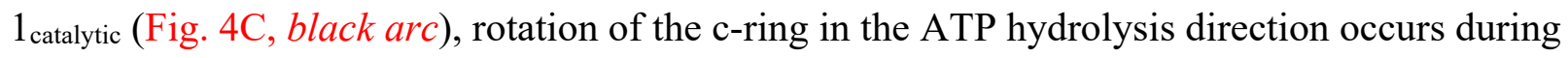
the transition from State $1_{\text {catalytic }}(\mathrm{h}) \rightarrow$ State $1_{\text {catalytic }}(\mathrm{a})$. As ATP hydrolysis in a catalytic $\alpha \beta_{\text {tight }}$ site causes the transition from a catalytic dwell to a binding dwell, the order of states indicates that the power stroke of ATP hydrolysis forces the peripheral stalk into a more strained conformation (e.g. State $1_{\text {catalytic }}(a) \rightarrow$ State $1_{\text {binding }}(d)$ ). This strain subsequently relaxes as the cring continues to turn in the ATP hydrolysis direction (e.g. State $1_{\text {binding }}(\mathrm{d}) \rightarrow$ State $1_{\text {binding }}(\mathrm{a})$ ). Conversely, rotation of the c-ring in the direction driven by proton translocation during ATP synthesis (Fig. 4C, counter-clockwise arrow) leads to increasing strain on the peripheral stalk (e.g. State $1_{\text {binding }}(\mathrm{a}) \rightarrow$ State $\left.1_{\text {binding }}(\mathrm{d})\right)$, which relaxes as ATP is formed in the catalytic site and the enzyme transitions from a binding dwell conformation to a catalytic dwell conformation (e.g. State $1_{\text {binding }}(\mathrm{d}) \rightarrow$ State $\left.1_{\text {catalytic }}(\mathrm{a})\right)$.

Notably, the most strained conformation of some of the states show less rotation of the c-ring in the ATP hydrolysis direction than less strained conformations of the preceding state (Fig. 4C, shaded areas). For example, the transition from State $1_{\text {catalytic }}(\mathrm{a})$ to State $1_{\text {binding }}(\mathrm{d})$ during ATP hydrolysis would involve the c-ring rotating $23^{\circ}$ in the ATP synthesis direction. The same apparent 'backstepping' can be seen at the transition from State 3 catalytic $\rightarrow$ State 3 binding, and State

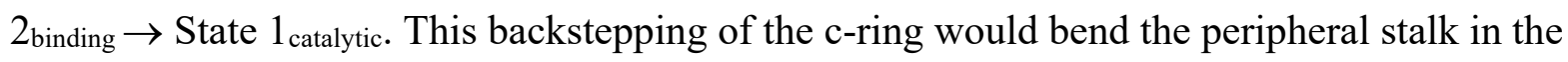
opposite direction of the applied force and is physically unlikely. Therefore, the unstrained conformations appear to show inactive complexes that are not part of the rotary sequence during substrate turnover. By extension, these data suggest that during rotary catalysis the peripheral stalk becomes strained and does not relax fully until catalysis stops. Construction of a movie showing rotation in the hydrolysis direction based on the most strained conformation of the enzyme illustrates the amount of deformation that can occur during ATP hydrolysis (Supplementary Video 2, 'ATP hydrolysis' cycle). Similarly, a video can be constructed showing rotation in the ATP synthesis direction based on the most strained conformations (Supplementary Video 2, 'ATP synthesis' cycle). Together, these data illustrate how in active ATP synthase the 
peripheral stalk can serve as a buffer that deforms under strain. In the fully active enzyme, the peripheral stalk likely remains deformed as the enzyme runs, with the degree of bending dependant on the rate of turnover, and with the enzyme only becoming fully relaxed in the absence of ATP or a proton motive force. 


\section{Methods}

Yeast growth and ATP synthase purification

Yeast was grown and ATP synthase was purified as described previously with minor modifications (Lau et al., 2008; Rubinstein et al., 2005). Briefly, yeast strain USY006 containing a $6 \times$ His tag at the $\mathrm{N}$ terminus of the $\beta$ subunits was grown in YPGD media $(1 \%[\mathrm{w} / \mathrm{v}]$ yeast extract, $2 \%[\mathrm{w} / \mathrm{v}]$ peptone, $3 \%[\mathrm{v} / \mathrm{v}]$ glycerol, $0.2 \%[\mathrm{w} / \mathrm{v}]$ glucose) with a $11 \mathrm{~L}$ fermenter $(\mathrm{New}$ Brunswick Scientific) for $\sim 48$ hours at $30{ }^{\circ} \mathrm{C}$ until saturation. All purification steps were performed at $4{ }^{\circ} \mathrm{C}$. Yeast cell walls were broken with bead beating, and cell debris was removed by centrifugation at $5,000 \times \mathrm{g}$ for $30 \mathrm{~min}$. Mitochondria were collected by centrifugation at $25,000 \times \mathrm{g}$ for $30 \mathrm{~min}$, before being washed with phosphate buffer $(50 \mathrm{mM}$ sodium phosphate $\mathrm{pH}$ 9.0, $5 \mathrm{mM}$ 6-aminocaproic acid, $5 \mathrm{mM}$ benzamidine, $1 \mathrm{mM}$ PMSF) for $30 \mathrm{~min}$. Washed mitochondria were collected by centrifugation at $184,000 \times \mathrm{g}$ for $30 \mathrm{~min}$, before being resuspended in buffer $(50 \mathrm{mM}$ Tris- $\mathrm{HCl} \mathrm{pH} 7.4,10 \%$ [v/v] glycerol, 1\% [w/w] DDM [Anatrace], $5 \mathrm{mM}$ 6-aminocaproic acid, $5 \mathrm{mM}$ benzamidine, $1 \mathrm{mM}$ PMSF) and solubilized with gentle shaking for one hour. Insoluble material was removed by centrifugation at $184,000 \times \mathrm{g}$ for 30 min, and supernatant containing solubilized protein was supplemented with $40 \mathrm{mM}$ imidazole and $300 \mathrm{mM} \mathrm{NaCl}$ before being loaded onto a $5 \mathrm{~mL}$ HisTrap column (Cytiva) equilibrated with HisTrap buffer $(50 \mathrm{mM}$ Tris- $\mathrm{HCl} \mathrm{pH} 7.4,10 \%$ [v/v] glycerol, 0.05\% [w/w], $40 \mathrm{mM}$ imidazole, $300 \mathrm{mM} \mathrm{NaCl}, 5 \mathrm{mM}$ 6-aminocaproic acid, $5 \mathrm{mM}$ benzamidine, $1 \mathrm{mM}$ PMSF) and washed with HisTrap buffer. ATP synthase was eluted with HisTrap buffer containing $300 \mathrm{mM}$ imidazole and was loaded onto a Superose 6 Increase column (Cytiva) equilibrated with buffer $(20 \mathrm{mM}$ Tris$\mathrm{HCl} \mathrm{pH} 7.4,10 \%$ [v/v] glycerol, 0.05\% [w/w] DDM, $100 \mathrm{mM} \mathrm{NaCl}, 5 \mathrm{mM} \mathrm{MgCl}$ ). Fractions containing ATP synthase were pooled, and the protein was concentrated to $\sim 15 \mathrm{mg} / \mathrm{ml}$ prior to cryoEM grid freezing or storage at $-80{ }^{\circ} \mathrm{C}$.

\section{CryoEM specimen preparation}

Glycerol in the ATP synthase preparation was removed with a Zeba Spin desalting column (Thermo Fisher Scientific [TFS]) before freezing cryoEM specimens. Holey gold films with $\sim 2$ $\mu \mathrm{m}$ holes were nanofabricated as described previously (Marr et al., 2014) on 300 mesh Maxtaform copper-rhodium grids (Electron Microscopy Sciences). Specimens with 10 mM ATP were prepared by first applying $0.4 \mu \mathrm{L}$ of $50 \mathrm{mM}$ ATP in buffer $(70 \mathrm{mM}$ Tris-HCl pH 7.4, 
$0.05 \%$ [w/w] DDM, $100 \mathrm{mM} \mathrm{NaCl}, 55 \mathrm{mM} \mathrm{MgCl}_{2}$ ) onto a grid that had been glow-discharged in air for $2 \mathrm{~min}$. Freshly prepared ATP synthase $(1.6 \mu \mathrm{L})$ was mixed quickly with the ATP solution on the grid before blotting for $1 \mathrm{~s}$ in an EM GP2 grid freezing device (Leica) at $4{ }^{\circ} \mathrm{C}$ and $100 \%$ humidity and plunge frozen in liquid ethane. Specimens without ATP were prepared the same way except that the mixing step was omitted.

\section{CryoEM data collection}

Preliminary cryoEM data was collected with FEI Tecnai F20 electron microscope operated and $200 \mathrm{kV}$ and equipped with a Gatan K2 Summit camera. Images with this microscope were acquired as movies with 30 fractions at 5 e/pixel/s and a calibrated pixel size of $1.45 \AA$ /pixel. CryoEM movies for high-resolution analysis were collected with a Titan Krios G3 microscope operated at $300 \mathrm{kV}$ and equipped with a Falcon 4 camera (TFS). Automated data collection was performed with $E P U$. For the dataset including ATP, 10,037 movies, each consisting of 30 fractions, were collected at a nominal magnification of $59,000 \times$, corresponding to a calibrated pixel size of $1.348 \AA$. The exposure rate and the total exposure of the specimen were $6.1 \mathrm{e}^{-}$ /pixel/s and $\sim 40 \mathrm{e}^{-} / \AA^{2}$, respectively. For the ATP-free dataset, 8,817 30-fraction movies were collected at a nominal magnification of $75,000 \times$, corresponding to a calibrated pixel size of $1.046 \AA$. The exposure rate and the total exposure for this specimen were $4.2 \mathrm{e} / \mathrm{pixel} / \mathrm{second}$ and $\sim 39 \mathrm{e} / \AA^{2}$, respectively.

\section{Image analysis}

Data collection was monitored with cryoSPARC Live (Punjani et al., 2017) to screen and select high-quality micrographs. All other image analysis steps were performed with cryoSPARC $v 2$ except where mentioned. Movie fractions were aligned with patch-based motion correction and contrast transfer function (CTF) parameters were estimated with patch-based CTF estimation. After removing movies with undesirable motion or CTF fit, 7,474 and 4,059 movies from the dataset including ATP and ATP-free dataset were selected for further processing, respectively. Movie fractions were aligned with MotionCor2 (Zheng et al., 2017) with a $7 \times 7$ grid and averaged micrographs from the aligned movies were subjected to patch-based CTF estimation. For the ATP-free dataset, particle selection was perform with Topaz (Bepler et al., 2019). For the dataset including ATP, templates for particle selection were generated from 2D classification of 
manually selected particle images. After particle selection, 2,534,488 particle images were extracted for the dataset with ATP and 442,025 particle images were extracted for the ATP-free dataset. Low quality particle images were removed with two rounds of 2D classifications, yielding 1,109,677 and 422,765 particle images for the dataset including ATP and the ATP-free dataset, respectively. Further cleaning with ab initio 3D classification and heterogeneous refinement reduced dataset sizes to 915,825 and 379,817 particle images, respectively. The remaining particle images were classified into three classes, corresponding to the three main rotational states of the enzyme, and each class was refined with non-uniform refinement (Punjani et al., 2020). For the dataset including ATP, local refinement was performed with all particle images with a mask including $\alpha_{3} \beta_{3} \gamma \delta \varepsilon$ from the $\mathrm{F}_{1}$ region. CTF parameters of individual particle images were re-estimated with local CTF refinement, and masked refinement was performed again with updated CTF parameters. Image alignment parameters were then converted to Relion (Scheres, 2012) .star file format with pyem (DOI: 10.5281/zenodo.3576630) and individual particle motion was corrected with Bayesian polishing (Zivanov et al., 2019). For the ATP-free dataset, Bayesian polishing was performed with an intact ATP synthase map reconstructed with all particle images and particle images were down-sampled to a pixel size of $1.308 \AA$. Motion corrected images were imported back to cryoSPARC, refined, and CTF parameters re-estimated. For the dataset including ATP, particle images were initially classified into four classes. Iterative $\mathrm{ab}$ initio classification and heterogeneous refinement of each of the four classes yielded 27 unique rotational states of ATP synthase. The 27 structures were named State $1_{\text {binding }}$ (a to d), State $1_{\text {catalytic }}\left(\mathrm{a}\right.$ to $\mathrm{h}$ ), State $2_{\text {binding }}$ (a to b), State $2_{\text {catalytic }}$ (a to e), State $3_{\text {binding }}$ (a to c), and State

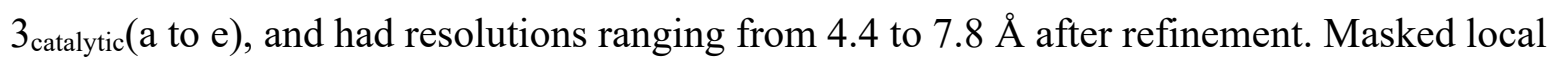

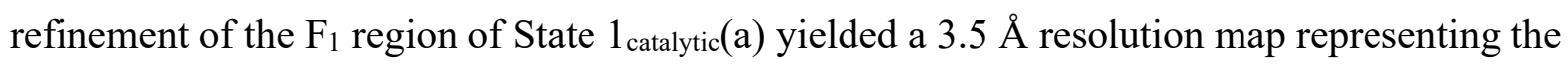
MgADP inhibited state. Local refinement of the $\mathrm{F}_{1}$ region of combined State $1_{\text {catalytic }}(\mathrm{e}$ to $\mathrm{h}$ ) and State $1_{\text {binding }}$ (a to d) yielded two $4.0 \AA$ resolution maps. To better visualize the nucleotide density in maps, density modification (Terwilliger et al., 2020) of locally refined maps of State

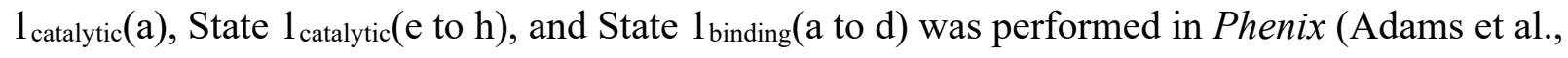
2010). For the ATP-free dataset, a similar $3 \mathrm{D}$ classification strategy yielded nine $\mathrm{F}_{1}$ states,

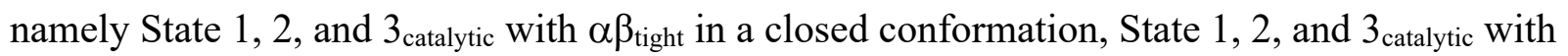
$\alpha \beta_{\text {tight }}$ in an open conformation, and State 1,2, and $3_{\text {binding with }} \alpha \beta_{\text {tight }}$ in an open conformation (Fig. S1, S3). These states included 56,739, 52,468, 24,879, 31,559, 47,065, 65,651, 19,922, 
57,468 and 23,622 particle images, respectively. Local refinement of the $F_{1}$ region with these images yielded maps at 3.4, 3.4, 3.6, 3.5, 3.5, 3.4, 3.7, 3.4, and $3.7 \AA$ resolution, respectively. When the three rotational states are combined, the $F_{1}$ regions of State catalytic $_{\text {with }} \beta_{\text {tight }}$ closed,

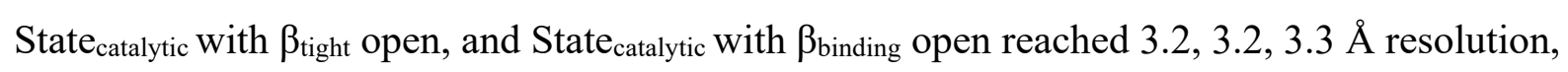
respectively. Classification of particle images contributing to State $1_{\text {catalytic }}$ from the ATP-free dataset yielded 4 classes with different c-ring positions relative to subunit a, which demonstrates the flexibility of the peripheral stalk in the absence of free ATP (Fig 1D, S1). Maps of these states were calculated from 41,506, 8,810, 10,550, and 1,984 particle images and reached 3.8, 4.4, 4.4, $7.1 \AA$ resolutions after refinement, respectively. A similar classification strategy was employed with the other two catalytic states, and particle images of the most relaxed State 1, 2, $3_{\text {catalytic(a) }}(191,939$ particle images) were used to calculate locally-refined maps of OSCP with its contact site on $\mathrm{F}_{1}$, the remainder of the peripheral stalk, and the $\mathrm{F}_{\mathrm{O}}$ region. These maps were combined with the map from local refinement of the $F_{1}$ region of the State $1_{\text {catalytic }}$ with $\alpha \beta_{\text {tight }}$ closed using the 'vop maximum' function in USCF Chimera to generate a composite map of the entire complex.

\section{Model building and refinement}

To build atomic models of the $F_{1}$ region, the crystal structure of yeast $F_{1}$ (PDB 2HLD)(Kabaleeswaran et al., 2006) was fitted as a rigid body into locally refined maps of $F_{1}$ in UCSF Chimera (Pettersen et al., 2004). Models were manually adjusted in Coot (Emsley and Cowtan, 2004) before being imported into ISOLDE (Croll, 2018) within ChimeraX (Goddard et al., 2018) to improve dihedral angles and rotamer fitting. A final round of refinement was performed with Phenix and the resulting models were evaluated with Molprobity (Chen et al., 2010) and EMRinger (Barad et al., 2015). To build backbone models of the full complex, a mosaic model was first assembled by rigid body fitting of the yeast $\mathrm{F}_{1}$ crystal structure (PDB 2HLD), subunits $a_{b c}$ dfi from the yeast $F_{O}$ cryoEM structure (PDB 6B2Z) (Guo et al., 2017), the peripheral stalk region without subunit $\mathrm{h}$ from the yeast monomer cryoEM structure (PDB 6CP3) (Srivastava et al., 2018), and domains of a subunit $\mathrm{h}$ atomic model predicted with AlphaFold (Jumper et al., 2021) into the unsharpened maps. Molecular Dynamics Flexible Fitting (Trabuco et al., 2008) was then performed for the 27 rotational states of the dataset 
including ATP to generate corresponding backbone models. Figures and movies were generated with ChimeraX and UCSF Chimera.

\section{Statement of contributions}

JLR conceived the project and supervised the research. HG purified the protein, performed the cryoEM, image analysis, and atomic model building. HG and JLR wrote the manuscript and prepared figures.

\section{Acknowledgements}

HG was supported by an Ontario Graduate Scholarship for International Students. JLR was supported by the Canada Research Chairs program. This work was supported by Canadian Institutes of Health Research grant PJT162186 (JLR). CryoEM data was collected at the Toronto High-Resolution High-Throughput cryoEM facility, supported by the Canada Foundation for Innovation and Ontario Research Fund.

Competing interests: The authors declare no competing interests.

Data availability: CryoEM maps are deposited in the Electron Microscopy Data Bank and atomic models are deposited in the Protein Data Bank with accession numbers indicated in Supplementary Tables 1 and 2. 

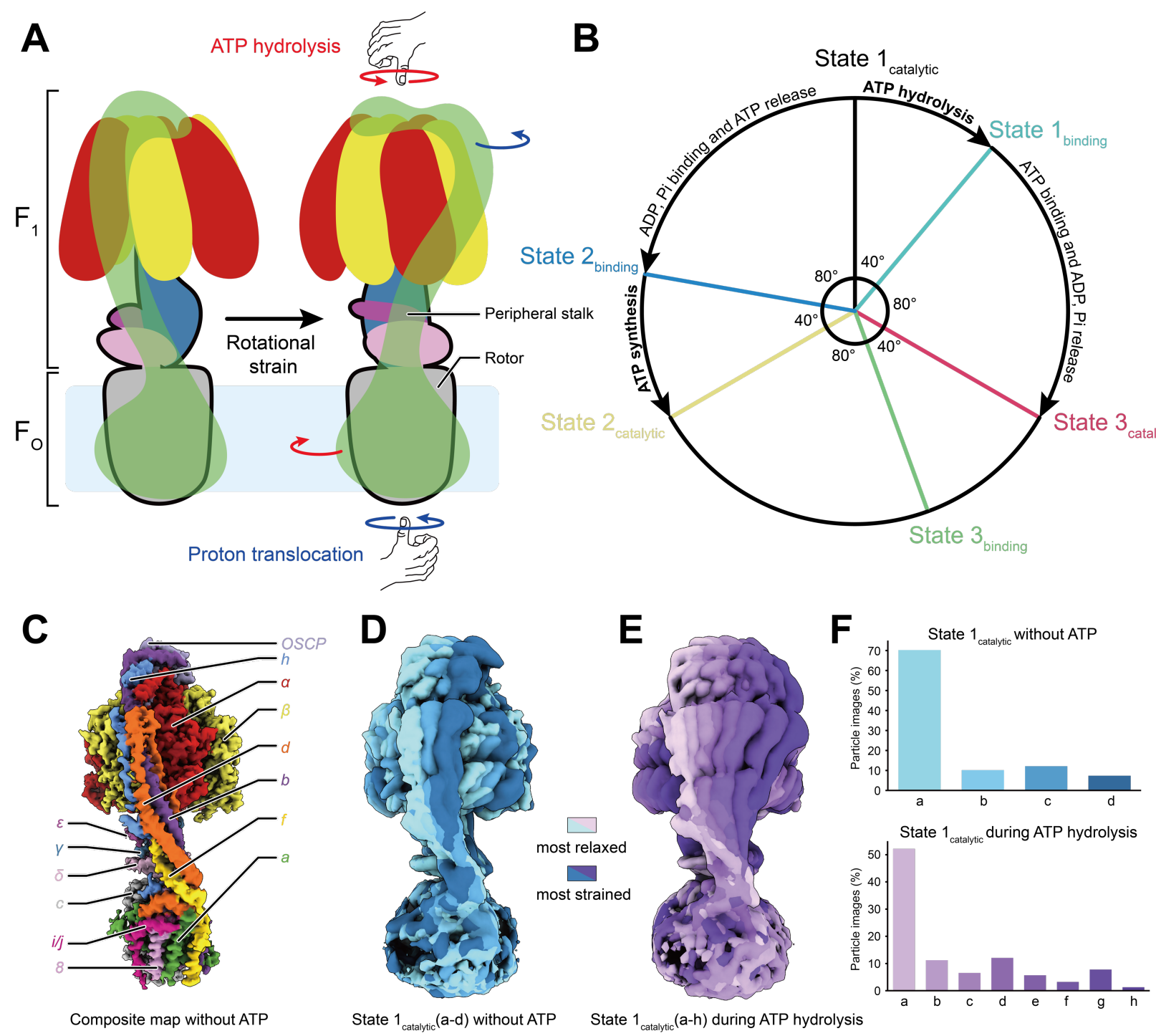

Figure 1. Rotation in ATP synthase. A, ATP synthase (left) consists of an $\mathrm{F}_{1}$ and an $\mathrm{F}_{\mathrm{O}}$ region with a shared rotor subcomplex (outlined in black) and a peripheral stalk (green). Rotation driven by proton translocation through the Fo region, or the opposite rotation driven by ATP hydrolysis in the $\mathrm{F}_{1}$ region, are predicted to induce a right-handed bend of the peripheral stalk (right). B, ATP hydrolysis or synthesis in the $\mathrm{F}_{1}$ region requires three catalytic and three bind dwell conformations. C, High-resolution structure of the yeast mitochondrial ATP synthase. D, In the absence of free ATP, the peripheral stalk exhibits limited flexibility with a left-handed curvature. E, During ATP hydrolysis, ATP synthase can adopt conformations that show a righthanded curvature of the peripheral stalk. F, Histograms of the distribution of conformations in the absence of ATP (top) and during ATP hydrolysis (bottom). 
A

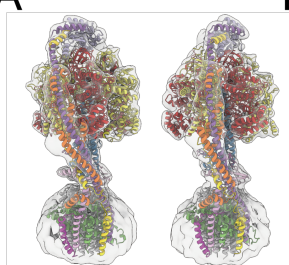

State $1_{\text {catalytic }}$ (a) State $1_{\text {catalytic }}$ (d)

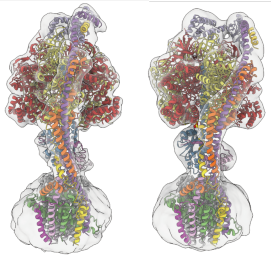

State $1_{\text {catalytic }}(\mathrm{f}) \quad$ State $1_{\text {catalytic }}(\mathrm{h})$
B

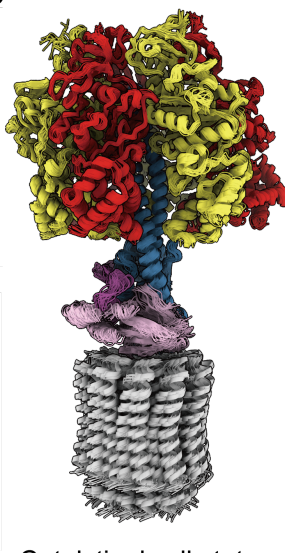

Catalytic dwell states

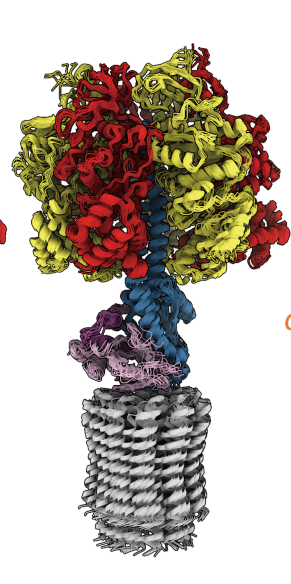

Binding dwell states

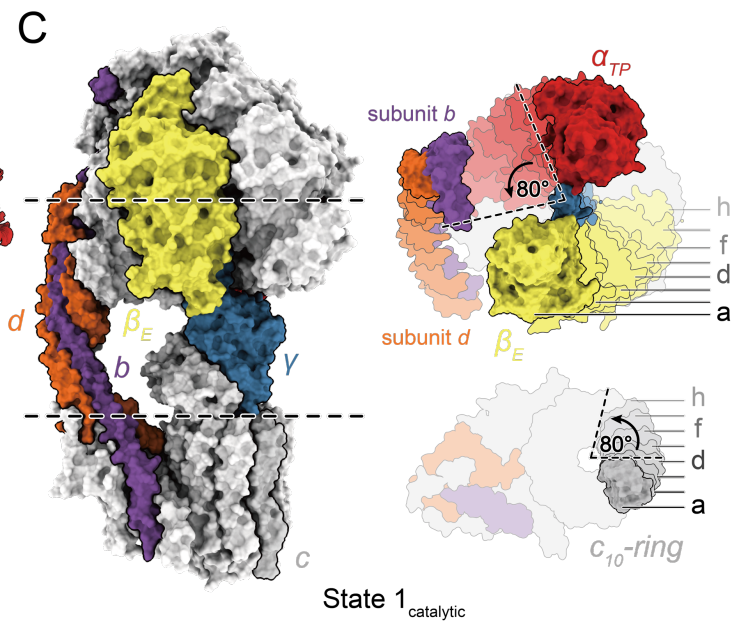

State $1_{\text {catalytic }}$

Figure 2. Atomic models of ATP synthase under strain during ATP hydrolysis. A, Atomic models of the enzyme were generated by flexible fitting into maps of the strained ATP synthase conformations. B, Overlay of fitted models from catalytic dwell (left) and binding dwell (right) structures show limited conformational changes in the $\alpha_{3} \beta_{3} \gamma \delta \varepsilon \varepsilon_{10}$ subcomplex. C, Overlay of models from catalytic dwell conformations show pronounced bending of the peripheral stalk while the enzyme is under strain during ATP hydrolysis. 

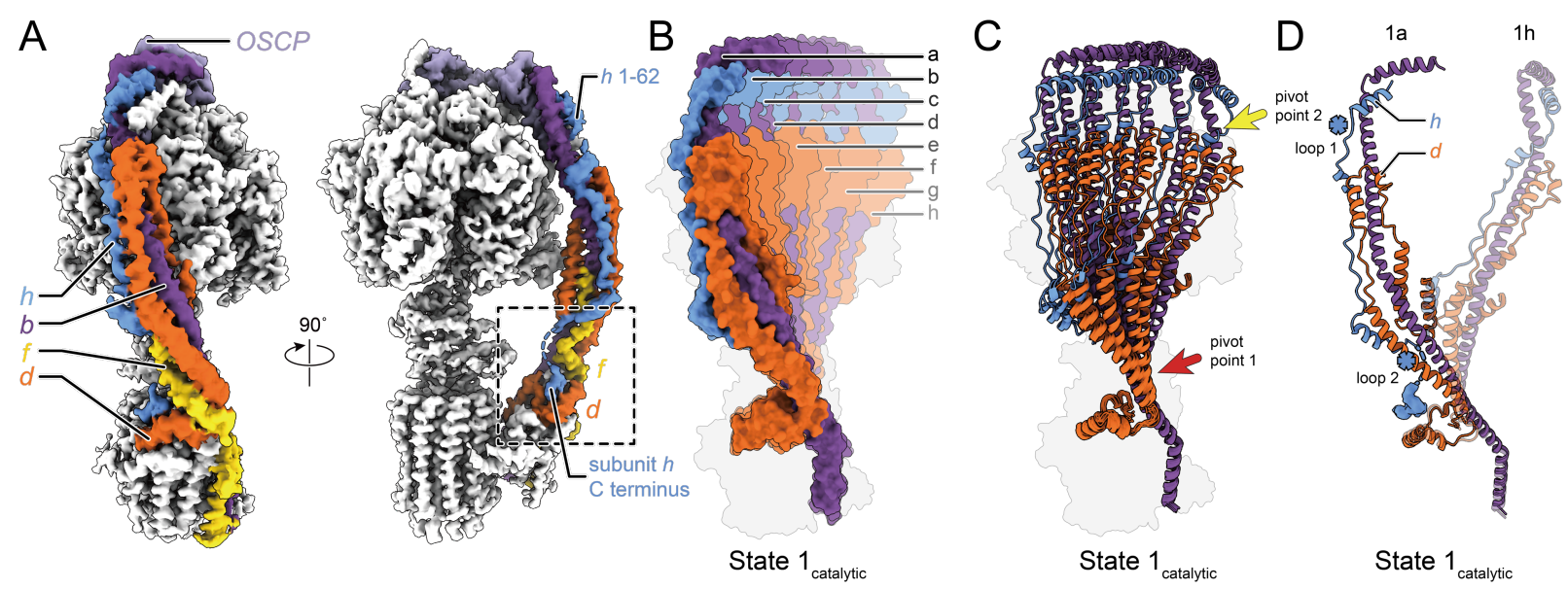

Figure 3. Deformation of the peripheral stalk of ATP synthase while under strain during ATP hydrolysis. A, Structure of the peripheral stalk shows that subunit $h$ bridges $F_{1}$ and $F_{O}$. B, Overlay of the eight State $1_{\text {catalytic }}$ conformations shows a large deformation of the peripheral stalk. C, The peripheral stalk bends at two pivot points near $\mathrm{F}_{1}$ (yellow arrow) and near $\mathrm{F}_{\mathrm{O}}$ (red arrow). D, Unstructured regions in subunit h (blue asterisks) allow it to withstand the bending of subunit d. 


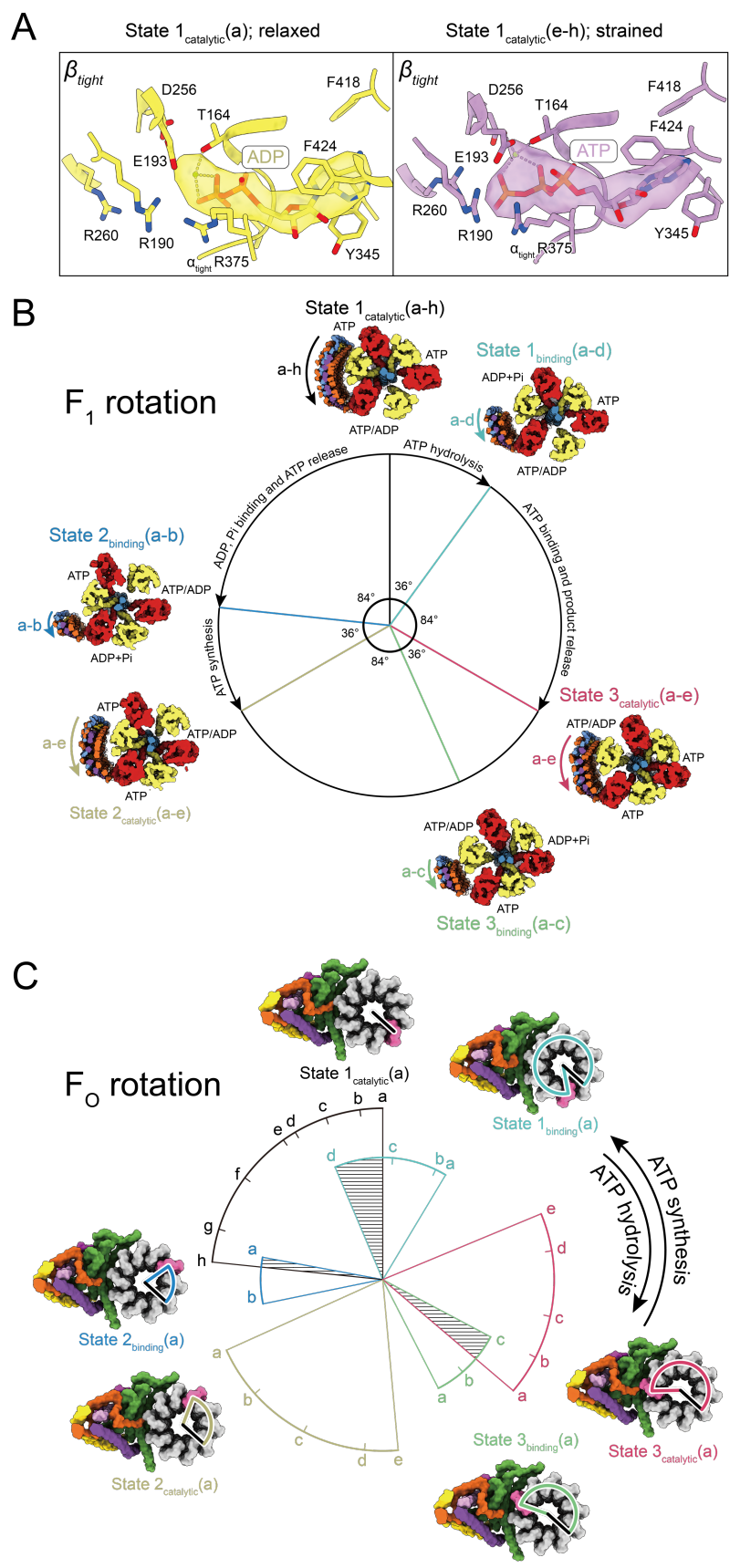

Figure 4. Sequence of conformations in the ATP hydrolysis and ATP synthesis cycles. A, The unstrained conformation of State $1_{\text {catalytic }}$ shows density consistent with MgADP in the $\alpha \beta_{\text {tight }}$ catalytic pair (left), suggesting an ADP-inhibited state, while the strained conformations show density consistent with MgATP (right) suggesting an active state. B, Measurement of the rotation of the rotor subunit $\gamma$ within the $\mathrm{F}_{1}$ region shows $36^{\circ}$ and $84^{\circ}$ sub-steps between catalytic and binding dwell conformations. $\mathbf{C}$, Measurement of the rotation of the c-ring relative to subunit a in $\mathrm{F}_{\mathrm{O}}$. Within each binding or catalytic dwell conformation the peripheral stalk becomes increasingly strained as the c-ring rotates in the ATP synthesis direction and increasingly relaxed as the c-ring rotates in the ATP hydrolysis direction. Transition between catalytic and binding dwell conformations would require back-stepping of the c-ring (shaded areas) if every conformation occurred during the rotary cycle. 


\section{References}

Abrahams, J.P., Leslie, A.G.W., Lutter, R., Walker, J.E., 1994. Structure at 2.8 Â resolution of F1ATPase from bovine heart mitochondria. Nature 370, 621-628. https://doi.org/10.1038/370621a0

Adachi, K., Oiwa, K., Nishizaka, T., Furuike, S., Noji, H., Itoh, H., Yoshida, M., Kinosita, K., 2007. Coupling of Rotation and Catalysis in F1-ATPase Revealed by Single-Molecule Imaging and Manipulation. Cell 130, 309-321. https://doi.org/10.1016/j.cell.2007.05.020

Adams, P.D., Afonine, P.V., Bunkóczi, G., Chen, V.B., Davis, I.W., Echols, N., Headd, J.J., Hung, L.W., Kapral, G.J., Grosse-Kunstleve, R.W., McCoy, A.J., Moriarty, N.W., Oeffner, R., Read, R.J., Richardson, D.C., Richardson, J.S., Terwilliger, T.C., Zwart, P.H., 2010. PHENIX: A comprehensive Python-based system for macromolecular structure solution. Acta Crystallogr. D Biol. Crystallogr. 66, 213-221. https://doi.org/10.1107/S0907444909052925

Barad, B.A., Echols, N., Wang, R.Y.R., Cheng, Y., Dimaio, F., Adams, P.D., Fraser, J.S., 2015. EMRinger: Side chain-directed model and map validation for 3D cryo-electron microscopy. Nat. Methods 12, 943-946. https://doi.org/10.1038/nmeth.3541

Bepler, T., Morin, A., Rapp, M., Brasch, J., Shapiro, L., Noble, A.J., Berger, B., 2019. Positive-unlabeled convolutional neural networks for particle picking in cryo-electron micrographs. Nat. Methods 16, 1153-1160. https://doi.org/10.1038/s41592-019-0575-8

Bilyard, T., Nakanishi-Matsui, M., Steel, B.C., Pilizota, T., Nord, A.L., Hosokawa, H., Futai, M., Berry, R.M., 2013. High-resolution single-molecule characterization of the enzymatic states in Escherichia coli F1-ATPase. Philos. Trans. R. Soc. B Biol. Sci. 368, 20120023. https://doi.org/10.1098/rstb.2012.0023

Bowler, M.W., Montgomery, M.G., Leslie, A.G.W., Walker, J.E., 2007. Ground state structure of F1ATPase from bovine heart mitochondria at 1.9 A resolution. J Biol Chem 282, 14238-14242. https://doi.org/M700203200 [pii] 10.1074/jbc.M700203200

Chen, V.B., Arendall, W.B., Headd, J.J., Keedy, D.A., Immormino, R.M., Kapral, G.J., Murray, L.W., Richardson, J.S., Richardson, D.C., 2010. MolProbity: All-atom structure validation for macromolecular crystallography. Acta Crystallogr. D Biol. Crystallogr. 66, 12-21. https://doi.org/10.1107/S0907444909042073

Cherepanov, D.A., Mulkidjanian, A.Y., Junge, W., 1999. Transient accumulation of elastic energy in proton translocating ATP synthase. FEBS Lett. 449, 1-6. https://doi.org/10.1016/S00145793(99)00386-5

Croll, T.I., 2018. ISOLDE: A physically realistic environment for model building into low-resolution electron-density maps. Acta Crystallogr. Sect. Struct. Biol. 74, 519-530. https://doi.org/10.1107/S2059798318002425

Emsley, P., Cowtan, K., 2004. Coot: Model-building tools for molecular graphics. Acta Crystallogr. D Biol. Crystallogr. 60, 2126-2132. https://doi.org/10.1107/S0907444904019158

Flygaard, R.K., Mühleip, A., Tobiasson, V., Amunts, A., 2020. Type III ATP synthase is a symmetrydeviated dimer that induces membrane curvature through tetramerization. Nat. Commun. 11. https://doi.org/10.1038/s41467-020-18993-6

Goddard, T.D., Huang, C.C., Meng, E.C., Pettersen, E.F., Couch, G.S., Morris, J.H., Ferrin, T.E., 2018. UCSF ChimeraX: Meeting modern challenges in visualization and analysis. Protein Sci. 27, 1425. https://doi.org/10.1002/pro.3235

Gu, J., Zhang, L., Zong, S., Guo, R., Liu, T., Yi, J., Wang, P., Zhuo, W., Yang, M., 2019. Cryo-EM structure of the mammalian ATP synthase tetramer bound with inhibitory protein IF1. Science 364, 1068-1075. https://doi.org/10.1126/science.aaw4852

Guo, H., Bueler, S.A., Rubinstein, J.L., 2017. Atomic model for the dimeric FO region of mitochondrial ATP synthase. Science 358, 936-940. https://doi.org/10.1126/science.aao4815 
Guo, H., Suzuki, T., Rubinstein, J.L., 2019. Structure of a bacterial ATP synthase. eLife 8, e43128e43128.

Hahn, A., Parey, K., Bublitz, M., Mills, D.J., Zickermann, V., Vonck, J., Kühlbrandt, W., Meier, T., 2016. Structure of a Complete ATP Synthase Dimer Reveals the Molecular Basis of Inner Mitochondrial Membrane Morphology. Mol. Cell 63, 445-456. https://doi.org/10.1016/j.molcel.2016.05.037

Hahn, A., Vonck, J., Mills, D.J., Meier, T., Kühlbrandt, W., 2018. Structure, mechanism, and regulation of the chloroplast ATP synthase. Science 360, eaat4318-eaat4318. https://doi.org/10.1126/science.aat4318

Hirono-Hara, Y., Noji, H., Nishiura, M., Muneyuki, E., Hara, K.Y., Yasuda, R., Kinosita, K., Yoshida, M., 2001. Pause and rotation of F(1)-ATPase during catalysis. Proc. Natl. Acad. Sci. U. S. A. 98 , 13649-54. https://doi.org/10.1073/pnas.241365698

Jumper, J., Evans, R., Pritzel, A., Green, T., Figurnov, M., Ronneberger, O., Tunyasuvunakool, K., Bates, R., Žídek, A., Potapenko, A., Bridgland, A., Meyer, C., Kohl, S.A.A., Ballard, A.J., Cowie, A., Romera-Paredes, B., Nikolov, S., Jain, R., Adler, J., Back, T., Petersen, S., Reiman, D., Clancy, E., Zielinski, M., Steinegger, M., Pacholska, M., Berghammer, T., Bodenstein, S., Silver, D., Vinyals, O., Senior, A.W., Kavukcuoglu, K., Kohli, P., Hassabis, D., 2021. Highly accurate protein structure prediction with AlphaFold. Nature 596, 583-589. https://doi.org/10.1038/s41586-021-03819-2

Junge, W., Lill, H., Engelbrecht, S., 1997. ATP synthase: an electrochemical transducer with rotary mechanics. Trends Biochem. Sci. 22, 420-423. https://doi.org/10.1016/S0968-0004(97)01129-8

Kabaleeswaran, V., Puri, N., Walker, J.E., Leslie, A.G.W., Mueller, D.M., 2006. Novel features of the rotary catalytic mechanism revealed in the structure of yeast F1 ATPase. EMBO J. 25, 54335442. https://doi.org/10.1038/sj.emboj.7601410

Kobayashi, R., Ueno, H., Li, C.-B., Noji, H., 2020. Rotary catalysis of bovine mitochondrial F 1 -ATPase studied by single-molecule experiments. Proc. Natl. Acad. Sci. 117, 1447-1456. https://doi.org/10.1073/pnas.1909407117

Lau, W.C.Y., Baker, L.A., Rubinstein, J.L., 2008. Cryo-EM structure of the yeast ATP synthase. J Mol Biol 382, 1256-1264. https://doi.org/S0022-2836(08)00994-7 [pii] 10.1016/j.jmb.2008.08.014

Liu, S., Charlesworth, T.J., Bason, J.V., Montgomery, M.G., Harbour, M.E., Fearnley, I.M., Walker, J.E., 2015. The purification and characterization of ATP synthase complexes from the mitochondria of four fungal species. Biochem. J. 468, 167-175. https://doi.org/10.1042/BJ20150197

Marr, C.R., Benlekbir, S., Rubinstein, J.L., 2014. Fabrication of carbon films with approximately $500 \mathrm{~nm}$ holes for cryo-EM with a direct detector device. J Struct Biol 185, 42-47. https://doi.org/10.1016/j.jsb.2013.11.002

Martin, J.L., Ishmukhametov, R., Hornung, T., Ahmad, Z., Frasch, W.D., 2014. Anatomy of F1-ATPase powered rotation. Proc. Natl. Acad. Sci. 111, 3715-3720. https://doi.org/10.1073/pnas.1317784111

Mühleip, A., Kock Flygaard, R., Ovciarikova, J., Lacombe, A., Fernandes, P., Sheiner, L., Amunts, A., 2021. ATP synthase hexamer assemblies shape cristae of Toxoplasma mitochondria. Nat. Commun. 12. https://doi.org/10.1038/s41467-020-20381-z

Mühleip, A., McComas, S.E., Amunts, A., 2019. Structure of a mitochondrial ATP synthase with bound native cardiolipin. eLife 8, 1-23. https://doi.org/10.7554/eLife.51179

Murphy, B.J., Klusch, N., Langer, J., Mills, D.J., Yildiz, Ö., Kühlbrandt, W., 2019. Rotary substates of mitochondrial ATP synthase reveal the basis of flexible F1-Fo. Science 364, eaaw9128. https://doi.org/10.1126/science.aaw9128

Nishizaka, T., Oiwa, K., Noji, H., Kimura, S., Muneyuki, E., Yoshida, M., Kinosita, K., 2004. Chemomechanical coupling in F1-ATPase revealed by simultaneous observation of nucleotide kinetics and rotation. Nat. Struct. Mol. Biol. 11, 142-148. https://doi.org/10.1038/nsmb721

Noji, H., Yasuda, R., Yoshida, M., Kinosita Jr., K., 1997. Direct observation of the rotation of F1ATPase. Nature 386, 299-302. https://doi.org/10.1038/386299a0 
Pänke, O., Rumberg, B., 1999. Kinetic modeling of rotary CF0F1-ATP synthase: storage of elastic energy during energy transduction. Biochim. Biophys. Acta BBA - Bioenerg. 1412, 118-128. https://doi.org/10.1016/S0005-2728(99)00059-6

Pettersen, E.F., Goddard, T.D., Huang, C.C., Couch, G.S., Greenblatt, D.M., Meng, E.C., Ferrin, T.E., 2004. UCSF Chimera - A visualization system for exploratory research and analysis. J. Comput. Chem. 25, 1605-1612. https://doi.org/10.1002/jcc.20084

Pinke, G., Zhou, L., Sazanov, L.A., 2020. Cryo-EM structure of the entire mammalian F-type ATP synthase. Nat. Struct. Mol. Biol. 27, 1077-1085. https://doi.org/10.1038/s41594-020-0503-8

Punjani, A., Rubinstein, J.L., Fleet, D.J., Brubaker, M.A., 2017. CryoSPARC: Algorithms for rapid unsupervised cryo-EM structure determination. Nat. Methods 14, 290-296. https://doi.org/10.1038/nmeth.4169

Punjani, A., Zhang, H., Fleet, D.J., 2020. Non-uniform refinement: adaptive regularization improves single-particle cryo-EM reconstruction. Nat. Methods 17, 1214-1221. https://doi.org/10.1038/s41592-020-00990-8

Rubinstein, J.L., Dickson, V.K., Runswick, M.J., Walker, J.E., 2005. ATP synthase from Saccharomyces cerevisiae: Location of subunit $\mathrm{h}$ in the peripheral stalk region. J. Mol. Biol. 345, 513-520. https://doi.org/10.1016/j.jmb.2004.10.060

Rubinstein, J.L., Walker, J.E., 2002. ATP synthase from Saccharomyces cerevisiae: location of the OSCP subunit in the peripheral stalk region. J Mol Biol 321, 613-619. https://doi.org/S002228360200671X [pii]

Rubinstein, J.L., Walker, J.E., Henderson, R., 2003. Structure of the mitochondrial ATP synthase by electron cryomicroscopy. EMBO J 22, 6182-6192. https://doi.org/10.1093/emboj/cdg608

Scheres, S.H.W., 2012. RELION: implementation of a Bayesian approach to cryo-EM structure determination. J Struct Biol 180, 519-530. https://doi.org/10.1016/j.jsb.2012.09.006

Sobti, M., Smits, C., Wong, A.S.W., Ishmukhametov, R., Stock, D., Sandin, S., Stewart, A.G., 2016. Cryo-EM structures of the autoinhibited E. coli ATP synthase in three rotational states. eLife 5, 1-18. https://doi.org/10.7554/eLife.21598

Sobti, M., Ueno, H., Noji, H., Stewart, A.G., 2021. The six steps of the complete F1-ATPase rotary catalytic cycle. Nat. Commun. 12, 4690. https://doi.org/10.1038/s41467-021-25029-0

Soga, N., Kimura, K., Kinosita, K., Yoshida, M., Suzuki, T., 2017. Perfect chemomechanical coupling of F o F 1 -ATP synthase. Proc. Natl. Acad. Sci. 114, 4960-4965. https://doi.org/10.1073/pnas. 1700801114

Sorgen, P.L., Bubb, M.R., Cain, B.D., 1999. Lengthening the Second Stalk of F1F0 ATP Synthase in Escherichia coli. J. Biol. Chem. 274, 36261-36266. https://doi.org/10.1074/jbc.274.51.36261

Sorgen, P.L., Caviston, T.L., Perry, R.C., Cain, B.D., 1998. Deletions in the Second Stalk of F1F0-ATP Synthase in Escherichia coli. J. Biol. Chem. 273, 27873-27878. https://doi.org/10.1074/jbc.273.43.27873

Spikes, T.E., Montgomery, M.G., Walker, J.E., 2020. Structure of the dimeric ATP synthase from bovine mitochondria. Proc. Natl. Acad. Sci. U. S. A. 117, 23519-23526. https://doi.org/10.1073/pnas.2013998117

Srivastava, A.P., Luo, M., Zhou, W., Symersky, J., Bai, D., Chambers, M.G., Faraldo-Gómez, J.D., Liao, M., Mueller, D.M., 2018. High-resolution cryo-EM analysis of the yeast ATP synthase in a lipid membrane. Science 360, 1-15. https://doi.org/10.1126/science.aas9699

Steel, B.C., Nord, A.L., Wang, Y., Pagadala, V., Mueller, D.M., Berry, R.M., 2015. Comparison between single-molecule and X-ray crystallography data on yeast F1-ATPase. Sci. Rep. 5, 8773. https://doi.org/10.1038/srep08773

Stock, D., Leslie, A.G., Walker, J.E., 1999. Molecular architecture of the rotary motor in ATP synthase. Science 286, 1700-1705. https://doi.org/10.1126/science.286.5445.1700

Terwilliger, T.C., Ludtke, S.J., Read, R.J., Adams, P.D., Afonine, P.V., 2020. Improvement of cryo-EM maps by density modification. Nat. Methods 17, 923-927. https://doi.org/10.1038/s41592-0200914-9 
Trabuco, L.G., Villa, E., Mitra, K., Frank, J., Schulten, K., 2008. Flexible Fitting of Atomic Structures into Electron Microscopy Maps Using Molecular Dynamics. Structure 16, 673-683. https://doi.org/10.1016/j.str.2008.03.005

Vik, S., Antonio, B.J., 1994. A mechanism of proton translocation by F1F0 ATP synthases suggested by double mutants of the a subunit. J Biol Chem 269, 30364-9.

Yasuda, R., Noji, H., Yoshida, M., Kinosita, K., Itoh, H., 2001a. Resolution of distinct rotational substeps by submillisecond kinetic analysis of F1-ATPase. Nature 410, 898-904. https://doi.org/10.1038/35073513

Yasuda, R., Noji, H., Yoshida, M., Kinosita, K., Itoh, H., 2001b. Resolution of distinct rotational substeps by submillisecond kinetic analysis of F1-ATPase. Nature 410, 898-904. https://doi.org/10.1038/35073513

Zheng, S.Q., Palovcak, E., Armache, J.P., Verba, K.A., Cheng, Y., Agard, D.A., 2017. MotionCor2: Anisotropic correction of beam-induced motion for improved cryo-electron microscopy. Nat. Methods 14, 331-332. https://doi.org/10.1038/nmeth.4193

Zhou, A., Rohou, A., Schep, D.G., Bason, J.V., Montgomery, M.G., Walker, J.E., Grigorieffniko, N., Rubinstein, J.L., 2015. Structure and conformational states of the bovine mitochondrial ATP synthase by cryo-EM. eLife 4, e10180-e10180. https://doi.org/10.7554/eLife.10180

Zivanov, J., Nakane, T., Scheres, S.H.W., 2019. A Bayesian approach to beam-induced motion correction in cryo-EM single-particle analysis. IUCrJ 6, 5-17. https://doi.org/10.1107/S205225251801463X 